\title{
The Maximum Principle for Control Distributions.
}

\author{
George K. Francis (Champaign-Urbana, Illinois, U.S.A.) (*)
}

\begin{abstract}
Summary. - Pontrjagin's maximum prineiple holds for a switchable distribution of $C^{1_{-} \text {regular }}$ nonautonomous vector fields on a finite dimensional $C^{2}$-manifold. The flow-theoretic proof does not require that the distribution contains those autonomous fields that correspond to constant controllers in the classical formulation.
\end{abstract}

\section{1. - Introduction.}

Many problems in the theory of optimal control have been advantageously reformulated in a geometrical setting. We follow the exposition of F. ALBRECHT [1, p. 27], but the reader may wish to refer also to $[6$, p. $36 ; 4$, p. 6]. Let $X$ be a differentiable manifold of class $C^{2}$, let $\Omega$ be a topological space, and let $\xi: X \times \Omega \rightarrow T X$ be a controllable vector field. That is, for each $\omega \in \Omega$, let $x \rightarrow \xi(x, \omega)$ be a $C^{1}$-vector field on the manifold (taking values in its tangent bundle $T X$ ) such that $\xi$, together with its partial differential with respect to $x$ varies continuously in $(x, \omega)$. The reader may think of $X$ as realizing the constraints on the state variables, and $\Omega$ as realizing the restraints on the control variables of a parametrized system $\xi$ of first order ordinary, not necessarily linear, differential equations. Let $\underline{\underline{U}}$ be a class of not necessarily continuous paths $u: I_{u} \rightarrow \Omega, I_{u}$ a compact real interval proper to the control $u$. For a given control $u \in U$ with $I_{u}=\left[t_{0}, t_{1}\right]$, a response at $x_{0} \in X$ is a curve $\alpha:\left[t_{0}, t^{*}\right] \rightarrow X, t_{0}<t^{*} \leqslant t_{1}$, such that $\alpha\left(t_{0}\right)=x_{0}$ and $\alpha^{\prime}(t)=\xi(\alpha(t), u(t))$ for almost every $t \in\left[t_{0}, t^{*}\right]$. Accordingly, we impose certain regularity conditions on the elements of $U$ in order for responses to exist, be unique, and to vary smoothly with the initial $\overline{\overline{d a}}$ ta, we define the attainable set $A\left(x_{0}, \xi, \underline{\underline{U}}\right)$ to be the entirety of all endpoints $\alpha\left(t^{*}\right)$ of responses to $\underline{\underline{U}}$ at $x_{0}$. If $X$ is finite dimensional and $\underline{\underline{U}}$ is closed under certain operations on its elements, a maximum principle, in the sense of Pontrjagin, gives necessary conditions for a response to terminate on the topological boundary of the attainabje set. The relation of such a response to an optimal solution of an associated problem of minimizing a certain cost functional is explained in $[1, \mathrm{p} .64 ; 6, \mathrm{p} .309]$.

Since a response at $x_{0}$ is in any case an integral curve of a nonautonomous dynamical system on $X, \dot{x}=\varphi(x, t)$ where $\varphi(x, t)=\xi(x, u(t))$, it is the philosophy of this paper to prove a maximum principle for a distribution $\Phi$ of time dependent

(*) Entrata in Redazione il 12 marzo 1972. 
vector fields $\varphi(x, t)$ on $X$ in terms of the flows generated by them. For this, a field $\varphi \in \Phi$ must satisfy certain regularity conditions (Section 2) for the flow to exist, and the family $\Phi$ must be closed under the switching operation (Section 3 ) for the maximum principle to hold.

The principal reason for dispensing with the notion of a control or "strategy" space $\Omega$ in this paper is notational economy. In a subsequent study [9], however, we shall show how a large class of control data $(X, \Omega, \xi, \underline{\underline{U}})$ and control distributions $(X, \Phi)$ may be canonically "embedded" in a "universal " control problem $(X, \Gamma X, e v, \underline{=})$, where $V$ is a switchable family of regulated paths in a Fréchet space $\Gamma X$ of vector fields on $X$ and $e v$ is the evaluation $\operatorname{map} X \times \Gamma X \rightarrow T X$.

Section 4 is devoted to our "flow theoretic" version of F. Albrecht's more "field theoretic" proof of the main theorem $[1$, pp. 56-63]. Our modification, and hopefully, simplification of his ingenious approach gains a modicum of generality by lowering the necessary class of $X$ from $C^{3}$ to $C^{2}$, and by avoiding the use of constant controllers entirely. Our exposition is cast in terms of the coordinate free calculus of Banach manifolds $[2,5,8]$ in order to pinpoint exactly the two places that finite dimensionality is required in the proof. (It is known that the maximum principle fails in infinite dimensional manifolds.)

\section{2. - Regular vector fields.}

This section serves to summarize known sufficient conditions for a time dependent vector field to generate local flows, and to introduce the notation that will be used in the remainder of this paper. Lemma A summarizes the context of regular vector fields of [1], which is based on Chapter $X$ of [2].

An abstract local flow on $X$ at $x_{0}$ may be defined as a smooth map $\mu: I \times J \rightarrow$ $\rightarrow C^{1}(U, X)$, where $I \times J \times U$ is open in $R \times R \times X$ and contains $\left(t_{0}, t_{0}, x_{0}\right)$, such that $\mu[t, t](x)=x$ for all $(t, x) \in(I \cap J) \times U$, and $\mu[s, t] \circ \mu[r, s]=\mu[r, t]$ wherever this expression is well defined. Note that if both $(s, t)$ and $(t, s)$ are in $I \times J$, then $\mu[s, t]$ is a diffeomorphism on a subneighborhood of $x_{0}$ inside $U$.

Recall $[2, \mathrm{p} .145]$ that a regulated path in a topological space is a function $p(t)$ from a real interval $I$ such that right (left) limits $\lim _{s \rightarrow 0^{+}} p(t \pm s)=p\left(t^{ \pm}\right)$exist whenever $t \pm s \in I$ for some small $s>0$. Recall $[1$, p. 35$]$ that a regulated path in a Banach space has at most countably many discontinuities.

Lemcra A. - Let $E$ be a Banach space, $I$ a real interval, $U$ open in $E$, and $f: U \times I \rightarrow E$ a function satisfying $[1, \mathrm{p}, 21]$ :

(H1) For every $t \in I, f_{t}(x)=f(x, t)$ is in $C^{1}(U, E)$,

(H2) $D_{x} f(x, t)$ is locally bounded on $U \times I$, and

(H3) for every $c \in C^{0}(I, U), f_{c}(t)=\left(f(c(t), t), D_{x} f(c(t), t)\right)$ is a regulated path in $E \times L(E)$, where $D_{x} f$ is the $x$-partial derivative of $f$ and $L(E)$ is the Banach space of continuous linear selfmaps of $E$. 
Then [1, pp. 7-12], on some (relatively) open neighborhood of $\left(x_{0}, t_{0}, t_{0}\right)$ in $U \times I \times I$ there exists the local flow $\mu=\int t$, where $\int f[s, t](x)=\alpha(t)$ satisfies $\alpha(s)=x$ and $\alpha^{\prime}(t)=f(\alpha(t), t)$ almost everywhere. The notation " $\int f$ » for the flow is suggestive of the integral equation

$$
\alpha(t)=x+\int_{s}^{t} f(\alpha(\tau), \tau) d \tau .
$$

Moreover, the differential $\int f[s, t](x)_{*}=D_{x}\left(\int f[s, t](x)\right)$ of the map $\int f[s, t]$ at $x$ satisfies the variational equation. That is, $\alpha_{*}(t)=\int f[s, t](x)_{*}$ satisfies $\alpha_{*}(s)=I_{E}$, the identity on $L(E)$, and for almost every $t$, the equation

$$
\alpha_{*}^{\prime}(t)=D_{x} f(\alpha(t), t) \cdot \alpha_{*}(t) \text {. }
$$

A field satisfying (H1-3) is called a regular vector field. The next lemma summarizes the process, described in Chapter IV of [5] for instance, of continuing solutions of an ordinary differential equation on a manifold along a particular integral curve. See also $[1$, pp. 12-26].

LEMMa B. - Let $X$ be a second countable Hausdorff manifold of class $C^{2}$ modelled on the separable Banach space $E$. Let $\varphi: X \times I_{\varphi} \rightarrow T X$ be a vector field for which Lemma A holds locally. That is, $h_{*} \circ \varphi \circ\left(h^{-1} \times i d\right): h(U) \times I \rightarrow E$ is regular, where $\left(h, h_{*}\right): T U \rightarrow h(U) \times E$ is a coordinate chart of $T X$. (If $\sigma \in C^{2}(R, U)$ has $\sigma(0)=x$ and $\sigma^{\prime}(0)=v \in T_{x} X$, recall that $h_{*}(v)=d h(\sigma(s)) /\left.d s\right|_{s=0}$ is an isomorphism of $T_{x} X$ with $E)$. Let $x_{0} \in X$ and $\left[t_{0}, t_{1}\right] \subset I$, be such that $\alpha(t)=\int \varphi\left[t_{0}, t\right]\left(x_{0}\right)$ exists for all $t \in\left[t_{0}, t_{1}\right]$. Then, there exists an open neighborhood $D_{0}$ of $x_{0}$, depending on $\left(\varphi, t_{0}, t_{1}\right)$, such that $\mu(t)=\int \varphi\left[t_{0}, t\right]$ is a $C^{1}$ diffeomorphism of $U_{0}$ into $X$ for each $t \in\left[t_{0}, t_{1}\right]$. Moreover, $\mu\left(t_{0}\right)(x)=x$ for all $x \in V_{0}$ and for almost every $t, D_{t} \mu(t)(x)=\varphi(\mu(t)(x), t)$. The continuous map $\left[t_{0}, t\right] \times T U \rightarrow T X$ given by $\mu(t)(x)_{*} \cdot v$, where $v \in T_{x} X$ and $\mu(t)(x)_{*}$ is the differential of $\mu(t)$ at $x$, reduces to the identity on $T_{x} X$ when $t=t_{0}$.

It should be pointed out that Dieudonnés condition (H3) of Lemma $A[2, p .303]$ has one surprisingly rich aspect we use later on. Let $E, G$ be a pair of normed linear spaces, $U$ open in $E, I$ a real interval and $f: U \times I \rightarrow G$ a function such that $f_{e}(t)=f(e(t), t)$ is a regulated path in $G$ for all $c \in C_{0}(I, U)$. A selection on $I$ shall be a function $e: I \rightarrow\{-1,0,+1\}$ such that $e(t) \neq+1(-1)$ at a right (left) end point of $I$, but otherwise arbitrary. A proper selection is one for which $e(t) \neq 0$ for any $t$. For a selection, define

$$
f^{e}(x, t)=\lim _{s \rightarrow 0+} f(x, t+e(t) s)= \begin{cases}f\left(x, t^{+}\right) & \text {if } e(t)=+1 \\ f(x, t) & \text { if } e(t)=0 \\ f\left(x, t^{-}\right) & \text {if } e(t)=-1,\end{cases}
$$

where the limits are taken along the constant path $c=x$. It is a consequence of Proposition 1, below, that this limit is independent of the path chosen. The existence 
of every $f\left(x, t^{+}\right)$is, however, insufficient to guarantee the existence of $f_{c}\left(t^{+}\right)$for every $c$. Take $f(x, t)=\operatorname{sgn}(x t)=-1,0,+1$ according as $x t$ is negative, zero or positive. While $f\left(x, t^{+}\right)$exists for all $(x, t), f\left(t \sin \left(t^{-1}\right), t\right)$ has no limit as $t \rightarrow 0^{+}$.

Proposition 1. - If $f$ is as above and $e\left(t_{0}\right)=+1$, then

$$
\lim f^{e}(x, t)=f\left(x_{0}, t_{0}^{+}\right) \quad \text { as }(x, t) \rightarrow\left(x_{0}, t_{0}\right) \text { and } 0 \leqslant+\left(t-t_{0}\right) .
$$

(The analogous assertion holds with + replaced by -.)

Proof. - Assume that, on the contrary, $\left(x_{n}, t_{n}\right) \rightarrow\left(x_{0}, t_{0}\right), t_{n} \geqslant t_{0}$, but that $\left|y_{n}-y_{0}\right|>\varepsilon>0$, where $y_{n}=f^{e}\left(x_{n}, t_{n}\right)$ and $y_{0}=f\left(x_{0}, t_{0}^{+}\right)$. Assume, without loss of generality, that the $t_{n}$ are weakly monotone decreasing and that the $x_{n}$ lie in a convex neighborhood of $x_{0}$. Recursively, choose small positive $\delta_{n}$ so that

$$
\left|f\left(x_{n}, s_{n}\right)-f^{e}\left(x_{n}, t_{n}\right)\right|<\frac{1}{2} \varepsilon, \quad \text { for } s_{n}=t_{n}+e\left(t_{n}\right) \delta_{n},
$$

and the $s_{n}$ are strictly monotone decreasing to $t_{0}$. Setting $r_{n}=\frac{1}{2}\left(s_{n}+s_{n+1}\right)$, construct the polygonal path $c \in C_{0}\left(\left[t_{0}, s_{1}\right], U\right)$ by

$$
c(t)=\left\{\begin{array}{ccc}
\frac{s_{n}-t}{s_{n}-r_{n}} x_{0}+\frac{t-r_{n}}{s_{n}-r_{n}} x_{n} & \text { if } \quad r_{n} \leqslant t \leqslant s_{n}, \\
\frac{r_{n}-t}{r_{n}-s_{n+1}} x_{n+1}+\frac{t-s_{n+1}}{r_{n}-s_{n+1}} x_{0} & \text { if } & s_{n} \leqslant t \leqslant r_{n}, \\
x_{0} & \text { if } t=t_{0} .
\end{array}\right.
$$

Continuity at $t_{0}$ follows from the fact that $\left|e(t)-x_{0}\right| \leqslant \sup \left|x_{0}-x_{n}\right|$ for all $t \leqslant s_{m}$. Set $y^{*}=f_{c}\left(t_{0}^{+}\right)$. Since $f_{c}\left(r_{n}\right)=f\left(x_{0}, r_{n}\right)$ and $r_{n} \rightarrow t_{0}^{+}$, it follows that $y^{*}=y_{0}$. Another subsequence is $f_{c}\left(s_{n}\right)=f\left(x_{n}, s_{n}\right)$, for which $\left|f_{c}\left(s_{n}\right)-y_{0}\right|>\frac{1}{2} \varepsilon$. Contradiction.

The hypothesis (H.1) in Lemma A, that the field be continuous let alone continuously differentiable in $x$, seems unnecessarily demanding, especially since the common assumption $[5, \mathrm{p}, 61]$ that $f$ be $O^{1}$ in both variables has already been liberalized by (H.2-3). Indeed, in a future study [9] we shall show that for a proper selection $e$, Proposition 1 implies that $f_{c}^{e}$ is again regulated for all $c$, and that $f^{e}(x, t)$ is continuous in $x$ and locally bounded in $(x, t)$. Further, replacing $D_{x} f$ in (H.3) by some weaker linear approximation than the Fréchet partial derivative, we shall demonstrate Lemma A for this properly larger class containing fields that violate (H.2) and hence (H.1).

\section{3. - Control distributions.}

For two time dependent vector fields $\varphi$ and $\psi$ on a manifold $X$ and real numbers, $r_{0} \leqslant r_{1}, s_{0} \leqslant s_{1}$, the field $\sigma$ is said to switch $\left(\varphi, r_{0}, r_{1}\right)$ to $\left(\psi, s_{0}, s_{1}\right)$, if $\left[r_{0}, r_{1}\right] \subset I_{\varphi}$, 
$\left[s_{0}, s_{1}\right] \subset I_{\psi}$, and for all $x \in X$,

$$
\varphi(x, t)= \begin{cases}\varphi\left(x, s_{0}+t-r_{1}\right) & \text { if } r_{1} \leqslant t \leqslant s_{1}+r_{1}-s_{0} \\ \varphi(x, t) & \text { if } r_{0} \leqslant t<r_{1},\end{cases}
$$

Note that if $r_{0}=r_{1}$, the first condition is vacuous. For a given $\varepsilon>0$, the switeh is said to be limited, advanced, retarded or exact, if $\left|s_{0}-r_{1}\right|<\varepsilon, r_{1} \leqslant s_{0}<r_{1}+\varepsilon$, $r_{1}-\varepsilon<s_{0} \leqslant r_{1}$ or $s_{0}=r_{1}$ respectively. Obviously, switching regular fields yields an regular field. A family of regular fields $\Phi$ which is closed under switching will be known as a control distribution on $X$. The attainable set $A\left(x_{0}, \Phi\right)$ is the set of end points $\varphi\left[t_{0}, t_{1}\right]\left(x_{0}\right)$, where $\varphi \in \Phi$ and $\left[t_{0}, t_{1}\right] \subset I_{\varphi}$.

We fix the symbols $x_{0}, \varphi$, and $t_{0}<t_{1}$, with

$$
\alpha(t)=\int \phi\left[t_{0}, t\right]\left(x_{0}\right) \quad \text { and } \quad \alpha_{*}(t)=\int \varphi\left[t_{0}, t\right]\left(x_{0}\right)_{*}
$$

There are two types of so called infinitesimal $\Phi$-alternative tangent vectors at $x$ associated with $\alpha$,

$$
\begin{aligned}
& v=\alpha_{*}(s)^{-1} \cdot \psi\left(\alpha(s), s^{+}\right), \\
& v=-\alpha_{*}(s)^{-1} \cdot \varphi\left(\alpha(s), s^{-}\right),
\end{aligned}
$$

(Type 2)

where $s \in\left[t_{0}, t_{1}\right] \cap I_{\psi}$ and $\psi \in \Phi$ for Type 1 , and $t_{0}<s \leqslant t_{1}$ for Type 2 .

THEonear 1. - If every vector in the tangent space $T_{0} X$ at $x_{0}$ is a finite nonnegative linear combination of a fixed finite collection of infinitesimal alternatives associated to the data $\left(\Phi, x_{0}, \varphi, t_{0}, t_{1}, \alpha\right)$ as above, then $x_{1}=\alpha\left(t_{1}\right)$ is an interior point of the attainable set $A\left(x_{0}, \Phi\right)$.

The integral path $\alpha$ is said to be $\Phi$-extremal if a certain adjoint or Hamiltonian equation has a maximal solution. With F. AlBkEoHT [1, p. 36] we express this in terms of a nontrivial continuous linear functional $h$ on $T_{0} X$ such that for almost every $t \in\left[t_{0}, t_{1}\right]$,

$$
\sup _{\psi \in \Phi} h\left(\alpha_{*}(t)^{-1} \cdot \psi(\alpha(t), t)\right)=h\left(\alpha_{*}(t)^{-k} \cdot \varphi(\alpha(t), t)\right)=0 .
$$

Precisely, if $e\left(\left(t_{0}, t_{1}\right]\right)=-1=-e\left(t_{0}\right)$, then for every $t$

$$
\sup _{\psi=\Phi} h\left(\alpha_{*}(t)^{-1} \cdot \psi\left(\alpha(t), t^{+}\right)\right)=h\left(\alpha_{*}(t)^{-1} \cdot \varphi^{e}(\alpha(t), t)\right)=0
$$

Roxin's theorem [7], asserting that once an integral path dips into the interior of the attainable set it remains therein, follows easily from the flow Lemma $B$.

We shall call $\alpha$ a $\Phi$-boundary, if $\alpha(t)$ lies in the topological boundary of $A\left(x_{0}, \Phi\right)$ for all $t \in\left[t_{0}, t_{1}\right]$. 
Theorem 2 (Pontrjagin's Maximum Principle). If $X$ is a finite dimensional manifolds then a $\Phi$-boundary is also a $\Phi$-extremal.

Proof. - Theorem 2 follows from the observation that if $\alpha$ is not extremal, then the elosure of the cone in $T_{0} X$ generated by the finite nonnegative linear combinations of the infinitesimal $\Phi$-alternatives fills out the entire tangent space $[1, p$. 37]. If $X$ is finite dimensional, the conditions of Theorem 1 may be met. Hence $\alpha\left(t_{1}\right)$ is not on $\operatorname{Bdy} A\left(x_{0}, \Phi\right)$ and $\alpha$ is not a $\Phi$-boundary.

\section{4. - Proof of theorem 1.}

Let $U=U\left(\varphi, t_{0}, t_{1}, x_{0}\right)$ be the neighborhood in Lemma $\mathrm{B}$ where the flow $\mu(t)=$ $=\int\left[t_{0}, t\right]$ takes its source. We shall assume that $U$ is a convex coordinate chart on the manifold and henceforth identify it with its image in $E$. For an infinitesimal alternative vector $v$ of Type 1 , there exists an $\varepsilon_{v}>0$ and a neighborhood $U_{v} \subset U$ of $x_{0}$, both depending on $s$ and $\psi$, such that the one-parameter family of diffeomorphisms,

$$
g(r)=\mu(s)^{-1} \circ \int \psi[s, s+r] \circ \mu(s),
$$

is well defined from $U_{v}$ into $U$ for $r \in\left[0, \varepsilon_{v}\right]$ and $[s, s+r] \subset I_{\psi}$. We shall call $g$ the alternative local flow for $v$. For a Type 2 alternative, this flow is given by

$$
g(r)=\mu(s)^{-1} \circ \mu(s-r),
$$

where, in addition, $\varepsilon_{v}<s-t_{0}$ must be assured.

Evidently, $g(0)$ is the identity on $U_{v}$, and its differential $g(0)(x)_{*}$ is the identity on $E$, for each $x \in U_{v}$, under the identification $T_{x} X=E$ we have made.

Proposition 2. - If $g$ is an alternative local flow for $v$, then for each $x \in U_{v}$ the curve $g_{x}(r)=g(r)(x)$ has, for almost every $r$, a tangent vector given by

$$
g_{x}^{\prime}(r)=\left\{\begin{array}{rc}
\mu(s)\left(g_{x}(r)\right)_{*}^{-1} \cdot \psi(\mu(s) \circ g(r)(x), s+r) & \text { (Type 1) } \\
-\mu(s)\left(g_{x}(r)\right)_{*}^{-1} \cdot \varphi(\mu(s) \circ g(r)(x), s-r) & \text { (Type 2). }
\end{array}\right.
$$

Moreover, $g^{\prime}\left(0^{+}\right)\left(x_{0}\right)=v$, and for $(x, w) \in U_{v} \times E$,

$$
\lim _{(r, s) \rightarrow\left(0^{+}, x_{0}\right)}\left(g(r)(x), g(r)(x) * \cdot w, g_{x}^{\prime}(r)\right)=\left(x_{0}, w, v\right) .
$$

Proof. - The computation of $g_{x}^{\prime}(r)$ follows immediately from the definition of the symbols and the chain rule. The continuous variation with respect to initial data of the solutions of the differential equations in Lemma $A$ and (H.3) suffice for the evaluation of $g^{\prime}\left(0^{+}\right)\left(x_{0}\right)$. 
Supplemented by the generosity of the limit in Proposition 1, this also suffices to establish $(4.5)$.

Let $v_{1}, v_{2}, \ldots, v_{n}$ be a finite number of infinitesimal alternatives, labelled in such a way that $s_{1} \leqslant s_{2} \leqslant \ldots \leqslant s_{n}$ and $s_{i-1}<s_{i}$ if $v_{i}$ is of Type 2. Choose common $\varepsilon_{0}>0$ and $U_{0} \subset U$ sufficiently small, such that $\varepsilon_{0}<s_{i}-s_{i-1}$ for all Type $2 v_{i}$, and that the composition

$$
G\left(r_{1}, r_{2}, \ldots, r_{n}\right)=g_{n}\left(r_{n}\right) \circ \ldots \circ g_{2}\left(r_{2}\right) \circ g_{1}\left(r_{1}\right)
$$

is well defined on $U_{0}$ with values in $U$ for all $\boldsymbol{r}=\left(r_{1}, r_{2}, \ldots, r_{n}\right) \in B^{n}\left(\varepsilon_{0}\right)=\left[0, \varepsilon_{0}\right]^{n}$ in $R^{n}$. Having identified $U$ with its $E$-image, we continue to denote the origin in $E$ by $x_{0}$ to distinguish it from $\mathbf{0}$, the origin in $R^{n}$. Let $F(\boldsymbol{r})=G(\boldsymbol{r})\left(x_{0}\right)$, then $F^{\prime}(\mathbf{0})=x_{0}$. Associated with the same set of infinitesimal alternatives is the linear map $L: R^{n} \rightarrow E: L(\boldsymbol{r})=\sum r_{i} v_{i}$. In Proposition 3 we show that $L$ is a sufficiently good linear approximation of $F$ so that (Proposition 4) $x_{0}$ is an interior point of $F\left(B^{n}\left(\varepsilon_{0}\right)\right)$ when $L$ maps the first orthant $R_{+}^{n}=\{\boldsymbol{r} \mid \boldsymbol{r} \geqslant 0\}$ onto $E$. (By abuse of notation, vector inequalities signify that the inequality holds for all components.) Finally, it remains to show (Proposition 5) that $\mu\left(t_{1}\right) \circ F\left(B^{n}\left(\varepsilon_{0}\right)\right) \subset A\left(x_{0}, \Phi\right)$ in order for $x_{1}=\mu\left(t_{1}\right)\left(x_{0}\right)$ to be an interior point of the attainable set.

Proposmon 3. - For $\boldsymbol{r}^{\prime}$ and $\boldsymbol{r}^{\prime \prime}$ in $B^{n}\left(\varepsilon_{0}\right)_{2}$

$$
\left|F\left(\boldsymbol{r}^{\prime \prime}\right)-F\left(\boldsymbol{r}^{\prime}\right)-L\left(\boldsymbol{r}^{\prime \prime}-\boldsymbol{r}^{\prime}\right)\right|=\left|\boldsymbol{r}^{\prime \prime}-\boldsymbol{r}^{\prime}\right| \theta\left(\boldsymbol{r}^{\prime}, \boldsymbol{r}^{\prime \prime}\right),
$$

where $\theta$ is a modulus of continuity vanishing as $\left(\boldsymbol{r}^{\prime}, \boldsymbol{r}^{\prime \prime}\right) \rightarrow(\mathbf{0}, \mathbf{0})$.

Proof. - Adding and subtracting terms, we see that LHS(4.6) $\leqslant\left|S_{1}\right|+\left|S_{2}\right|+$ $+\ldots+\left|S_{n+1}\right|$, where

$$
S_{i}=F\left(r_{1}^{\prime}, \ldots, r_{i-1}^{\prime}, r_{i}^{\prime \prime}, \ldots, r_{n}^{\prime \prime}\right)-F\left(r_{1}^{\prime}, \ldots, r_{i}^{\prime}, r_{i+1}^{\prime \prime}, \ldots, r_{n}^{\prime \prime}\right)-\left(r_{i}^{\prime \prime}-r_{i}^{\prime}\right) v_{i} .
$$

For a fixed index $i$ let us abbreviate

$$
\boldsymbol{u}=\left(r_{1}^{\prime}, \ldots, r_{i-1}^{\prime}, 0, \ldots, 0\right) \quad \text { and } \quad \boldsymbol{w}=\left(0, \ldots, 0, r_{i+1}^{\prime \prime}, \ldots, r_{n}^{\prime \prime}\right) \text {, }
$$

and, without loss of elarity, drop the subscript $i$. Thus

$$
\begin{aligned}
S=G(\boldsymbol{w}) \circ g\left(r^{\prime \prime}\right) \circ F(\boldsymbol{u})-G(\boldsymbol{w}) \circ g\left(r^{\prime}\right) \circ F(\boldsymbol{u})-\left(r^{\prime \prime}-r^{\prime}\right) v= \\
=\int_{r^{\prime}}^{r^{\prime \prime}}\left\{G(\boldsymbol{w})(g(r) \circ F(\boldsymbol{u}))_{*} \cdot g^{\prime}(r)(F(\boldsymbol{u}))\right\} d r-\left(r^{\prime \prime}-r^{\prime}\right) g^{\prime}\left(0^{+}\right)\left(x_{0}\right)= \\
=\int_{r^{\prime}}^{\tau^{\prime \prime}}\left\{G(\boldsymbol{w})(g(r) \circ F(\boldsymbol{u}))_{*} \cdot g^{\prime}(r)(F(\boldsymbol{u}))-G(\mathbf{0})\left(g(0) \circ F^{\prime}(\mathbf{0})\right)_{*} \cdot g^{\prime}\left(0^{+}\right)(F(\mathbf{0}))\right\} d r .
\end{aligned}
$$

8 - Anali di Matematica 
Hence, by Proposition 2, $|\boldsymbol{S}|=\left|r^{\prime \prime}-r^{\prime}\right| \theta\left(r^{\prime}, r^{\prime \prime}, \boldsymbol{u}, \boldsymbol{w}\right)$. But then, each $\boldsymbol{S}_{i}$ is surely $\left|\boldsymbol{r}^{\prime \prime}-\boldsymbol{r}^{\prime}\right| \theta\left(\boldsymbol{r}^{\prime}, \boldsymbol{r}^{\prime \prime}\right)$.

Proposition 4. - Let $F: B^{n}\left(\varepsilon_{0}\right) \rightarrow E$ be any function for which $F(\mathbf{0})=x_{0}$ and $L$ is any linear map for which $L\left(R_{+}^{n}\right)=E$. If $(4.6)$ holds, then $x_{0}$ is an interior point of $F\left(B^{n}\left(\varepsilon_{0}\right)\right)$.

Proof. - Note that, by hypothesis, $E$ is finite dimensional. Let $\boldsymbol{e}=(1,1, \ldots, 1)$ and solve $L(\boldsymbol{r})=-L(\boldsymbol{e})$ with $\boldsymbol{r} \geqslant 0$. Then $L(\boldsymbol{r}+\boldsymbol{e})=x_{0}$ and $\boldsymbol{r}+\boldsymbol{e}>0$. Hence the kernel of $L$ is at least one dimensional, and so extends into $R_{+}^{n}$. If

$$
k>\max _{|x|=1} \min \left\{|\boldsymbol{r}|: \boldsymbol{r} \in L^{-1}(x) \cap R_{+}^{n}\right\}
$$

then for every $x \in E$ and $x \neq x_{0}$ we can solve $L(\boldsymbol{r})=x$ such that $0<\boldsymbol{r}<k|x|$.

Now choose $\delta, 0<\delta<\varepsilon_{0}$, sufficiently small so that $\theta$ is exceeded by $1 / 2 k$ on $B^{n}(\delta)$. We propose to show that for every $x_{*} \in E$ with $\left|x_{*}\right|<\delta / 2 k, x_{*}=F\left(\boldsymbol{r}_{*}\right)$ for some $\boldsymbol{r}_{*}$. Recursively construct a sequence of points $\boldsymbol{r}_{n} \in B^{n}(\delta)$ such that either the construction stops with some $F\left(\boldsymbol{r}_{n}\right)=x_{0}$, or that

$$
0<\boldsymbol{r}_{n}-\boldsymbol{r}_{n-1}<\delta / 2^{n}
$$

and

$$
\left|x_{n}\right|<\delta / k 2^{n+1}
$$

where

$\left(\mathrm{III}_{n}\right)$

$$
x_{n}=x_{*}-F\left(\boldsymbol{r}_{n}\right) \neq x_{0} .
$$

For $n=1$, either $x_{*}=x_{0}$, and the construction stops with $\boldsymbol{r}_{1}=\boldsymbol{r}_{*}=0$, or $x_{*} \neq x_{0}$. Set $\boldsymbol{r}_{0}=\mathbf{0}$ and solve $L\left(\boldsymbol{r}_{1}\right)=x_{*}$ with $0<\boldsymbol{r}_{1}<k\left|x_{*}\right|<\delta / 2$. Hence $\left(\mathrm{i}_{1}\right)$ holds. For (iii $\left.{ }_{1}\right)$, let $x_{1}=x_{*}-F\left(\boldsymbol{r}_{1}\right)$ and for $\left(\mathrm{ii}_{1}\right)$, compute

$$
\left|F\left(\boldsymbol{r}_{1}\right)-x_{*}\right|=\left|F\left(\boldsymbol{r}_{1}\right)-F\left(\boldsymbol{r}_{0}\right)-L\left(\boldsymbol{r}_{1}-\boldsymbol{r}_{0}\right)\right|<(\delta / 2)(1 / 2 k) .
$$

Inductively, if $x_{n}=x_{0}$ then $\boldsymbol{F}\left(\boldsymbol{r}_{n}\right)=x_{*}$. Otherwise, $x_{n} \neq x_{0}$ and we solve $L(\boldsymbol{r})=x_{n}$ with $0<\boldsymbol{r}<k\left|x_{n}\right|<\delta / 2^{n+1}$ by $\left(\mathrm{ii}_{n}\right)$. Set $\boldsymbol{r}_{n+1}=\boldsymbol{r}+\boldsymbol{r}_{n}$ to obtain $\left(i_{n+1}\right)$.

Furthermore, from $\left(i_{j}\right), j \leqslant n$, we have that

$$
\begin{aligned}
& 0<\boldsymbol{r}_{n}=\left(\boldsymbol{r}_{n}-\boldsymbol{r}_{n-1}\right)+\ldots+\left(\boldsymbol{r}_{1}-\boldsymbol{r}_{0}\right)<\left(\delta / 2^{n}+\ldots+\delta / 2\right)<\delta, \\
& 0<\boldsymbol{r}_{n+1}=\boldsymbol{r}+\boldsymbol{r}_{n}<\delta / 2^{n+1}+\delta / 2^{n}+\ldots+\delta / 2<\delta .
\end{aligned}
$$

Hence

$$
\left|F\left(\boldsymbol{r}_{n+1}\right)-F\left(\boldsymbol{r}_{n}\right)-L\left(\boldsymbol{r}_{n+1}-\boldsymbol{r}_{n}\right)\right|<\left|\boldsymbol{r}_{n+1}-\boldsymbol{r}_{n}\right| 2 z^{2}
$$


Setting $x_{n+1}=x^{*}-F\left(\boldsymbol{r}_{n+1}\right)$ and recalling $\left(\mathrm{iii}_{n}\right)$, we have $\operatorname{LHS}(4.7)=\left|-x_{n+1}\right|$. By $\left(\mathrm{i}_{n}\right)$, RHS $(4.7)<\delta / k 2^{n+1}$. Hence $\left(\mathrm{ii}_{n+1}\right)$ holds and the recursion is complete.

Unless the process ceases at $\boldsymbol{F}\left(\boldsymbol{r}_{n}\right)=x_{*}$, we have by (i), a Cauchy sequence $\boldsymbol{r}_{n}$ in $B^{n}(\delta)$ that converges, say to $\boldsymbol{r}_{*}$. By (ii), the sequence $x_{n}$ in $E$ converges to the origin $x_{0}$. Since $F$ is continuous in $B^{n}(\delta)$ for sufficiently small $\delta, F\left(\boldsymbol{r}_{*}\right)=x_{*}$ by (iii).

Proposition 5. - Returning to the notation of the beginning of this section,

$$
\mu\left(t_{1}\right)(F(\boldsymbol{r})) \in A\left(x_{0}, \Phi\right), \quad \text { for } \boldsymbol{r} \in B^{n}\left(\varepsilon_{0}\right) .
$$

Proof. - Set $s_{0}=t_{0}$ and $s_{n+1}=t_{1}$. We recursively show

$$
\mu\left(s_{i}\right) \circ F\left(r_{1}, \ldots, r_{i}, 0, \ldots, 0\right) \in A\left(x_{0}, \Phi\right), \quad \text { for } i=0,1, \ldots, n+1
$$

For $i=0$,

$$
\mu\left(s_{0}\right) \circ F(0, \ldots, 0)=\int \varphi\left[t_{0}, t_{0}\right]\left(x_{0}\right)=x_{0} .
$$

Inductively, suppose that

$$
\mu\left(s_{i-1}\right) \circ F\left(\ldots, r_{i-1}, 0, \ldots\right)=\int \sigma_{i-1}\left[t_{0}, q_{i-1}\right]\left(x_{0}\right)
$$

for some $\sigma_{i-1} \in \Phi$ and $q_{i-1} \geqslant t_{9}$. For $v_{i}$ of Type 1 ,

$$
\begin{aligned}
\mu\left(s_{i}\right) \circ F\left(\ldots, r_{i}, 0, \ldots\right) & =\int \psi_{i}\left[s_{i}, s_{i}+r_{i}\right] \circ \mu\left(s_{i}\right) \circ F\left(\ldots, r_{i-1}, 0, \ldots\right) \\
& =\int \psi_{i}\left[s_{i}, s_{i}+r_{i}\right] \circ \int \varphi\left[s_{i-1}, s_{i}\right] \circ \int \sigma_{i-1}\left[t_{0}, q_{i-1}\right]\left(x_{0}\right)=\int \sigma_{i}\left[t_{0}, q_{i}\right]\left(x_{0}\right),
\end{aligned}
$$

where $\sigma_{i}$ is obtained by switching $\sigma_{i-1}$ to $\varphi$ and then to $\psi_{i}$, and $q_{i}=q_{i-1}+r_{i}+s_{i}-s_{i-1}$. If $v_{i}$ is of Type 2, we have that

$$
\begin{aligned}
\mu\left(s_{i}\right) \circ F\left(\ldots, r_{i}, 0, \ldots\right)=\mu\left(s_{i}-r_{i}\right) \circ F\left(\ldots, r_{i-1}, 0, \ldots\right) & \\
& =\int \varphi\left[s_{i-1}, s_{i}-r_{i}\right] \circ \int \sigma_{i-1}\left[t_{0}, q_{i-1}\right]\left(x_{0}\right)=\int \sigma_{i}\left[t_{0}, q_{i}\right]\left(x_{\theta}\right),
\end{aligned}
$$

where $\sigma_{i}$ is $\sigma_{i-1}$ switehed to $\varphi$ and $q_{i}=q_{i-1}-r_{i}+s_{i}-s_{i-1}$.

In the last step,

$$
\mu\left(t_{1}\right) \circ F\left(r_{1}, \ldots, r_{n}\right)=\int \varphi\left[s_{n}, t_{1}\right] \circ \int \sigma_{n}\left[t_{0}, q_{n}\right]\left(x_{0}\right)=\int \sigma\left[t_{0}, q\right]\left(x_{0}\right),
$$

where $\sigma$ is $\sigma_{n}$ switched to $\varphi$ and

$$
q=t_{1}-\Sigma(-1)^{T(i)} r_{i}, \quad \text { where } v_{i} \text { has type } T(i) .
$$

Note that the switching required to construct $\sigma$ is $n \varepsilon_{0}$-limited. 


\section{5. - Application.}

It is clear from (4.8) that $I_{\sigma}=\left[t_{0}, q\right]$, but, in general, we cannot expect that $q=t_{1}$ : Thus, to prove a maximum principle for the time-dependent or "small " attainable set

$$
A\left(x_{0}, \Phi, t_{0}, t_{1}\right)=\left\{\int \varphi\left[t_{0}, t_{1}\right]\left(x_{0}\right): \varphi \in \Phi \text { and }\left[t_{0}, t_{1}\right] \subset I_{\varphi}\right\},
$$

the argument may be modified as follows.

Proposition 6. - If $x_{1}=\alpha\left(t_{1}\right)=\int \varphi\left[t_{0}, t_{1}\right]\left(x_{0}\right)$ lies on the boundary of $A\left(x_{0}, \Phi, t_{0}, t_{1}\right)$ then there is a nontrivial linear functional $h$ on $T_{0} X$ such that for all $t \in\left(t_{0}, t_{1}\right)$

$$
\sup _{\psi \neq i} h\left(\alpha_{*}(t)^{-1} \cdot \psi\left(\alpha(t), t^{+}\right)\right)=h\left(\alpha_{*}(t)^{-1} \cdot \varphi\left(\alpha(t), t^{-}\right)\right) .
$$

Proof, - Consider a set of $n=2 m$ infinitesimal alternatives $v_{i}$ such that $s_{2 j}=s_{2 j+1}, v_{2 j}$ is of Type 2 and $v_{2 j+1}$ of Type $1, j=1, \ldots, m$. Let us write

$$
s_{z j}=s_{2 j+1}=s_{j}^{\prime} \quad \text { and } \quad A: R^{m} \rightarrow R^{n}: A\left(\mathbf{r}^{\prime}\right)=\boldsymbol{r},
$$

for the linear embedding $r_{2 j}=r_{2 j+1}=r_{j}^{\prime}$. Note that $A\left(B^{m}\left(\varepsilon_{0}\right)\right) \subset B^{n}\left(\varepsilon_{0}\right)$. Hence $L^{\prime}=L \circ \Lambda$ and $F^{\prime}=F \circ A$ are well defined, and Propositions 3 and 4 hold with $m$ replaeing $n$. Applying Proposition 5 to this data yields $q=t_{1}$ because $T(2 j)=$ $=1+T(2 j+1)$. We note that if $v_{2 j+1}=\alpha_{*}\left(s_{j}^{\prime}\right)^{-1} \cdot \psi_{i}\left(\alpha\left(s_{j}^{\prime}\right), s_{j}^{\prime+}\right)$, the switching step becomes

$$
\int \sigma_{j}\left[t_{0}, s_{j}^{\prime}\right]=\int \psi_{j}\left[s_{j}^{\prime}, s_{j}^{\prime}+r_{j}^{\prime}\right] \circ \varphi\left[s_{j-1}^{\prime}, s_{j}^{\prime}-r_{j}^{\prime}\right] \odot \sigma_{j-1}\left[t_{0}, s_{j-1}^{\prime}\right]\left(x_{0}\right) .
$$

Now consider the cone $K$ in the $T_{0} X$ generated by vectors

$$
w=a_{*}(s)^{-1} \cdot \psi\left(\alpha(s), s^{+}\right)-\alpha_{k}(s)^{-1} \cdot \varphi\left(\alpha(s), s^{-}\right) \text {, }
$$

where $s \in\left(t_{0}, t_{1}\right) \cap I_{\psi}$ and $\psi \in \Phi$. If $K \neq T_{0} X$ then the functional for a supporting hyperplane has $\sup h(w)=0$, and hence (5.1) follows. On the other hand, if $K=T_{0} X$, it remains to find a finite set of generators $w_{j}$ of Type 3 with distinct $s_{j}$. If we let the vector $w$ defined above vary with $s$, then $\lim _{\sigma \rightarrow s} w(\sigma)=w(s)$. (This follows from the continuity of $\alpha$ and $\alpha_{*}$ in $s$ and the limit (2.5)). Consequently, if the $w_{i}$ span $T_{0} X$ but some $s_{j}=s_{i+1}$, we may replace $w_{j+1}$ by a nearby $w_{j+1}^{\prime}$ with $s_{j+1}^{\prime}>s_{i j}$

Recall the closure operations on the family of controllers of $[1, p .28]$, which we translate into the notation of fields:

(C.1) For $(\varphi, s) \in \Phi \times I_{\varphi}$, the autonomous vector field $\psi(x, t)=\varphi(x, s)$ for all $t \in I_{\varphi}$, is in $\Phi$. 
(C.2) For $\varphi \in \Phi$, the restriction of $\varphi$ to a subinterval of $I_{\varphi}$ is in $\Phi$.

(C.3) For $(\varphi, s) \in \Phi \times R$, the $s$-shift, $\varphi_{s}(x, t)=\varphi(x, t-s)$ for all $t \in I_{\varphi}+s$, is in $\Phi$.

(C.4) The exact switch of two $\Phi$ fields is in $\Phi$.

(C.5) The zero field belongs to $\Phi$.

Note that closure under (C.2-4) implies closure under switching (3.1). Conversely, trivial switching $\left(r_{1}=s_{0}=s_{1}\right)$ restricts $\varphi$ to $\left[r_{0}, r_{1}\right]$. Advanced switching ean be interpreted as an intermediate switch to the zero field on $\left[r_{1}, s_{0}\right]$, and retarded switching is the exact switeh of $\varphi$ to the $\left(r_{1}-s_{0}\right)$-shift of $\psi$. Thus the only essential difference is the absence of (C.1), which corresponds to constant controllers. This omission is motivated by a control situation in which every attempt at holding a controller constant fails due to "drifting " of the equipment.

On the other hand, we have had to keep limited inexact switching ((C.3) and $\left.|s| \leqslant n \varepsilon_{0}\right)$ for Proposition 5, and arbitrary shifting is required for transitivity: $x_{1} \in A\left(x_{0}\right) \Rightarrow A\left(x_{1}\right) \subset A\left(x_{0}\right)$. Indeed, exact switehing ((C.2) and (C.4)) alone does not guarantee the maximum principle. For example, let $\Phi_{0}$ be the distribution generated by the single field in $R^{2}, \varphi\left(x_{1}, x_{2}, t\right)=\left(x_{1}, 6 x_{1} t\right), I_{\varphi}=[0,1]$, under exact switching only. Then $\Phi_{0}$ consists only in $\varphi$ and its restrictions. Hence $\alpha(t)=\left(t, 2 t^{3}\right)$ is a $\Phi_{0}$-boundary, $x_{0}$ the origin. It is not an extremal, because $\alpha_{*}(t)^{-1} \cdot \varphi(\alpha(t), t)=$ $=\left(1,3 t^{2}\right)$. Hence, the Type 1 alternatives at $t=0$ and 1 and the Type 2 alternative at $t=\frac{1}{2}$ span the plane under nonnegative linear combination.

From the flow theoretic point of view, an autonomous (in fact, a piecewise autonomous) field is freely shiftable in the sense that the integral depends on the length but not location of the interval. Thus we observe that (5.2) can be achieved by exact switching if $\psi_{i}$ is piecewise autonomous. Hence Proposition 6 holds also if we exchange (O.1) for (O.3) in our notion of switching. This is a partial answer to the question raised by H. HALKIN [3] whether shifting can be avoided. We shall go deeper into this question in a subsequent paper.

We wish to thank Prof. F. AuBrechT for his indispensible criticism and patient reading of several drafts of this paper, which began as a scholium to this masterful work $[1]$.

\section{REFERENCES}

[1] F. AlBRECHT, Topics in Control Theory, Springer, New York, 1968.

[2] J. Dieudonnté, Foundations in Modern Analysis, 2nd Edition, Academic Press, New York, 1969.

[3] G. Francis, The Pontrjagin-Albrecht maximum principle, presented to the Control Theory Conference of the Society for Natural Philosophy, Ann Arbor, 1969. 
[4] H. HALKIN, On the necessary condition for optimal control of nonlinear systems, J. Anal. Math., 12 (1963), pp. 1-82.

[5] S. LaNG, Introduction to Differentiable Manifolds, Interscience Publishers, New York, 1962.

[6] E. Lex - L. Markus, Foundationl of Optimal Control Theory, John Wiley and Sons, New York, 1967.

[7] E. RoxiN, A geometric interprelation of Pontrjagin's maximum principle, in: Nonlinear Differential Equations and Nonlinear Mechanics, Academic Press, New York, 1963.

[8] J. Schwartz, Nonlinear Functionat Analysis, notes by H. Fatrorini, R. Nirenberg and H. Ponta, Courant Institute, New York, 1965.

[9] G. FRANCIs, Cauonical representation of cerlain optimal control problems, to appear in J. Math. Anal. Applie. (1973). 